\title{
Hardy-Sobolev Derivatives of Phase and Amplitude, and Their Applications ${ }^{1}$
}

\author{
Pei Dang ${ }^{\mathrm{a}}$, Tao Qian ${ }^{2 b}$ \\ ${ }^{a}$ Department of Mathematics, University of Macau, Macao (Via Hong Kong). Email: \\ ya77408@umac.mo \\ ${ }^{b}$ Department of Mathematics, University of Macau, Macao (Via Hong Kong). Email: \\ fsttq@umac.mo
}

\begin{abstract}
In time-frequency analysis there are fundamental formulas expressing the mean and variance of the Fourier frequency of signals, $s$, originally defined in the Fourier frequency domain, in terms of integrals against the density $|s(t)|^{2}$ in the time domain. In the literature the existing formulas are only for smooth signals, for it is the classical derivatives of the phase and amplitude of the signals that are involved. The two representations of the covariance also rely on the classical derivatives and thus are restrictive. In this fundamental study, by introducing a new type of derivatives, called Hardy-Sobolev derivatives, we extend the formulas to signals in the Sobolev space that do not usually have classical derivatives. We also investigate the corresponding formulas for periodic (infinite discrete) and finite discrete signals.
\end{abstract}

Keywords: amplitude-phase representation of signal, Hilbert transform, instantaneous frequency, derivatives of phase and amplitude, Sobolev space, Hardy space

\section{Introduction}

Analytic instantaneous frequency of a real- or complex-valued signal $s(t)$ is defined to be $\varphi^{\prime}(t)$, where $\varphi(t)$ is the phase function of the quadrature amplitude-phase representation of the associated (complex-valued) analytic signal As(t) $=s(t)+i H s(t)=\rho(t) e^{i \varphi(t)}, \rho(t)=|A s(t)|$, where $H$ stands for the Hilbert transformation (see (1.9)). Besides the analytic instantaneous frequency one also studies the quadrature instantaneous frequency of a real- or complex-valued signal that is the derivative $\varphi^{\prime}(t)$ in the quadrature amplitude-phase representation of the original signal $s(t)=\rho(t) e^{i \varphi(t)}, \rho(t)=|s(t)|$. Since the classical derivative $\varphi^{\prime}(t)$ may not exist, the two types of instantaneous frequencies have not yet been well defined. Through the Hardy-Sobolev spaces decomposition the present paper presents a new type of derivatives for signals in the Sobolev space that generalizes the classical derivatives.

\footnotetext{
${ }^{1}$ The work was partially supported by Macao Sci. and Tech. Develop. Fund 014/2008/A1 and University of Macau Research Grant CG071/08-09S/QT/FST.

${ }^{2}$ Corresponding author. Tel: (+853) 8397 4954, Fax: (+853) 28838314 ,

Department of Mathematics, University of Macau, Macao (Via Hong Kong). Email: fsttq@umac.mo.
} 
Throughout this study we concern four types of signals: continuous signals $s(t)$, continuous periodic signals $s\left(e^{i t}\right)$, infinite discrete signals $\{x(n)\}_{n=-\infty}^{+\infty}$ and finite (periodic) discrete signals $\{x(n)\}_{n=0}^{N-1}$. Assume all the signals we study are of unit energy. We start with the classical derivatives setting. Let $s(t)=\rho(t) e^{i \varphi(t)} \in L^{2}(\mathbb{R}), \rho(t)=|s(t)|$. Assume that the classical derivatives $\rho^{\prime}(t), \varphi^{\prime}(t)$ and $s^{\prime}(t)$ exist for almost all $t \in \mathbb{R}$ as Lebesgue measurable functions, and $s^{\prime}$ is in $L^{2}(\mathbb{R})$. Then there hold

$$
\langle\omega\rangle=\int_{-\infty}^{\infty} \varphi^{\prime}(t)|s(t)|^{2} d t
$$

and

$$
\sigma_{\omega}^{2}=\int_{-\infty}^{\infty} \rho^{\prime 2}(t) d t+\int_{-\infty}^{\infty}\left(\varphi^{\prime}(t)-\langle\omega\rangle\right)^{2} \rho^{2}(t) d t
$$

where $\langle\omega\rangle$ is the mean of the Fourier frequency defined by

$$
\langle\omega\rangle \triangleq \int_{-\infty}^{\infty} \omega|\hat{s}(\omega)|^{2} d \omega
$$

and $\sigma_{\omega}^{2}$ is the bandwidth defined by

$$
B^{2}=\sigma_{\omega}^{2} \triangleq \int_{-\infty}^{\infty}(\omega-\langle\omega\rangle)^{2}|\hat{s}(\omega)|^{2} d \omega=\left\langle\omega^{2}\right\rangle-\langle\omega\rangle^{2}
$$

(see [5]), where $\hat{s}(\omega)$ is the Fourier transform of $s(t)$ (see $(2.14)$ ).

The proofs of the above mentioned results heavily depend on the differentiation rules of products and compositions of differentiable functions. A general signal $s \in L^{2}(\mathbb{R})$ cannot be expected to have classical derivatives $s^{\prime}, \rho^{\prime}$ and $\varphi^{\prime}$ as measurable functions with the desired integrability properties. Without those derivatives, however, the above representation formulas and relations are meaningless. It is natural to ask, if the signal understudy is not differentiable, then what will happen to these formulas.

In order to extend the formulas (1.1), and (1.2) to non-smooth signals, we work on the Sobolev space $L_{1}^{2}(\mathbb{R})$ and adopt the Hardy-Sobolev decomposition. The following results are established in [6]. If $s(t)$ and its distributional derivative $s^{\prime}(t)$ both belong to $L^{2}(\mathbb{R})$, i.e. $s \in L_{1}^{2}(\mathbb{R})$, then

$$
\begin{gathered}
\langle\omega\rangle=\int_{-\infty}^{\infty} \varphi^{+^{\prime}}(t) \rho^{+^{2}}(t) d t+\int_{-\infty}^{\infty} \varphi^{-\prime}(t) \rho^{-2}(t) d t \\
B^{2}=\int_{-\infty}^{\infty}\left[\left(\rho^{+^{\prime}}(t)\right)^{2}+\left(\rho^{-\prime}(t)\right)^{2}\right] d t+\int_{-\infty}^{\infty}\left[\left(\varphi^{+^{\prime}}(t)-\langle\omega\rangle\right)^{2} \rho^{+^{2}}(t)+\left(\varphi^{-\prime}(t)-\langle\omega\rangle\right)^{2} \rho^{-2}(t)\right] d t
\end{gathered}
$$


where

$$
\varphi^{ \pm \prime}(t) \triangleq \operatorname{Im}\left[\frac{s^{ \pm^{\prime}}(t)}{s^{ \pm}(t)}\right], \quad \rho^{ \pm^{\prime}}(t) \triangleq \rho^{ \pm}(t) \operatorname{Re}\left[\frac{s^{ \pm^{\prime}}(t)}{s^{ \pm}(t)}\right], \quad \text { a.e. }
$$

and

$$
\rho^{ \pm}(t) \triangleq\left|s^{ \pm}(t)\right|
$$

where $s^{ \pm}(t)$ are defined through the Hardy spaces decomposition $s=s^{+}+s^{-}, s^{ \pm}=(1 / 2)(s \pm$ $i H s), H$ is the Hilbert transformation, defined by

$$
H s(t) \triangleq \frac{1}{\pi} \lim _{\epsilon \rightarrow 0} \int_{|t-u|>\epsilon} \frac{s(u)}{t-u} d u \text {. }
$$

The functions $s^{ \pm}$are, respectively, the boundary values of the analytic functions

$$
s^{ \pm}(z)=\frac{ \pm 1}{2 \pi i} \int_{-\infty}^{\infty} \frac{s(u)}{u-z} d u=\frac{1}{\sqrt{2 \pi}} \int_{-\infty}^{\infty} e^{i t \omega} \chi_{ \pm}(\omega) e^{-y \omega} \hat{s}(\omega) d \omega, \quad z=t+i y \in \mathbb{C}^{ \pm}
$$

where $\chi_{ \pm}=\chi_{\mathbb{R}^{ \pm}}, \mathbb{R}^{+}=(0,+\infty)$ and $\mathbb{R}^{-}=(-\infty, 0)$, and, in general, $\chi_{E}$ is the characteristic function of the Lebesgue measurable set $E$ that takes value 1 on $E$ and 0 otherwise, and $s^{ \pm^{\prime}}(t)$ are the non-tangential boundary limits of $s^{ \pm^{\prime}}(z) \in H^{2}\left(\mathbb{C}^{ \pm}\right)$, the latter being the Hardy spaces for the upper- and lower-half of the complex plane, respectively, namely

$$
s^{ \pm^{\prime}}(t)=\lim _{z \rightarrow t} s^{ \pm^{\prime}}(z), \quad z \in \mathbb{C}^{ \pm}
$$

In the same way, we generalize the formulas for the mean of time, $\langle t\rangle$, and the duration, $\sigma_{t}^{2}$. The above formulas are all composed of two parts of which one corresponds to $s^{+}(t)$, and the other to $s^{-}(t)$.

The idea of the Hardy decomposition is based on the concept "analytic signal" originated by Gabor [8]. The topic of analytic signal is concerned by a great amount of literature, including $[17,11,5,18,19]$, that are, in particular, related to the concept "instantaneous frequency". The relations between the "instantaneous frequency" and Fourier frequency for signals in the continuous case are examined in [5, 7, 14]. In [21], the authors study these relations for the discrete case where signals and their spectra both are assumed to be periodic.

Both the Hardy and Sobolev spaces are classical topics of long histories of development with ample applications in the practical and theoretical sciences. The Hardy-Sobolev spaces in recent years have undergone a new phase of development $([1,13])$. To the authors' knowledge, apart from the proceeding study [6], no applications in signal analysis have been noted.

In [6] we show that under the classical derivatives assumptions the formulas (1.5) and (1.6), being valid for functions in the Sobolev space, are reduced to the classical formulas (1.1) and (1.2). It can be immediately seen that if $s=s^{+}\left(s^{-}=0\right.$, such signals are regarded as "onesided"), then the formulas (1.5) and (1.6) are also reduced to (1.1) and (1.2). In the present paper we introduce a new type of derivatives, called Hardy-Sobolev derivatives, based on which 
we obtain formulas (1.1) and (1.2) in terms of the new type of derivatives, being valid for general signals $s$ in the Sobolev space. In other words, the classical formulas become valid for non-smooth and two-sided signals (i.e. $s^{+} \neq 0, s^{-} \neq 0$ ), if we replace the classical derivatives by the Hardy-Sobolev derivatives. The latter are given by

Definition 1.1. If $s(t)=\rho(t) e^{i \varphi(t)} \in L_{1}^{2}(\mathbb{R})$, then we define the Hardy-Sobolev derivatives of $s(t), \rho(t)$ and $\varphi(t)$ by, respectively,

$$
\begin{gathered}
s^{*}(t)=s^{+^{\prime}}(t)+s^{{ }^{\prime}}(t), \\
\rho^{*}(t)= \begin{cases}\rho(t) \operatorname{Re}\left[\frac{s^{{ }^{\prime}}(t)+s^{-\prime}(t)}{s^{+}(t)+s^{-}(t)}\right] & \text { if } s(t) \neq 0, \\
0 & \text { if } s(t)=0,\end{cases}
\end{gathered}
$$

and

$$
\varphi^{*}(t)= \begin{cases}\operatorname{Im}\left[\frac{s^{+^{\prime}}(t)+s^{-^{\prime}}(t)}{s^{+}(t)+s^{-}(t)}\right] & \text { if } s(t) \neq 0, \\ 0 & \text { if } s(t)=0\end{cases}
$$

where $s^{ \pm}(t)$ are defined by (1.10). In the sequel, "Hardy-Sobolev derivative" is abbreviated as "H-S derivative". It is obvious that $s^{*}, \rho^{*} \in L^{2}(\mathbb{R})$ and $\varphi^{*}$ is measurable.

In $\S 2$, by using the $\mathrm{H}-\mathrm{S}$ derivatives and the related techniques we show that the formulas (1.5) and (1.6) return to their classical forms (1.1) and (1.2) with the derivatives $\varphi^{\prime}, \rho^{\prime}$ being replaced by $\varphi^{*}, \rho^{*}$. Moreover we further prove the equivalence between the two representations of the covariance of time and frequency. $\S 3$ studies these formulas for periodic signals and infinite discrete signals. The setting for the mean of the time $t$ and the related variance is motivated by [21]. For completeness $\S 4$ cites the similar relations for finite discrete-time signals.

\section{Means of Frequency and Time, and Covariance in Terms of H-S Derivatives}

The Fourier transform of $s \in L^{1}(\mathbb{R})$ is defined by

$$
\hat{s}(\omega) \triangleq \frac{1}{\sqrt{2 \pi}} \int_{-\infty}^{\infty} e^{-i t \omega} s(t) d t .
$$

If $\hat{s}$ is also in $L^{1}(\mathbb{R})$, then the inversion formula holds, that is

$$
s(t)=\frac{1}{\sqrt{2 \pi}} \int_{-\infty}^{\infty} e^{i t \omega} \hat{s}(\omega) d \omega, \quad \text { a.e. }
$$

There holds the Plancherel Theorem

$$
\|\hat{s}\|_{2}^{2}=\|s\|_{2}^{2}, \quad s \in L^{1}(\mathbb{R}) \cap L^{2}(\mathbb{R}) .
$$

Both the Fourier transformation and its inverse can be extended to $L^{2}(\mathbb{R})$ in which the Plancherel Theorem remains to hold. In the case the formulas (2.14) and (2.15) are valid in the $L^{2}$-sense. 
We adopt the notation $L_{n}^{2}(\mathbb{R})$ for the Sobolev spaces $([20])$, that is

$$
L_{n}^{2}(\mathbb{R})=\left\{s(t) \in L^{2}(\mathbb{R}): s^{(n)}(t) \in L^{2}(\mathbb{R})\right\}
$$

with the norm defined by

$$
\sqrt{\|s\|_{2}^{2}+\left\|s^{(n)}\right\|_{2}^{2}}
$$

where $s^{(n)}(t)$ denotes the square-integrable $n$-th distributional derivative of $s$. In this work we are concerned with only $L_{1}^{2}(\mathbb{R})$.

In [6], for $s \in L_{1}^{2}(\mathbb{R})$, we discussed five types of derivatives:

(i) the distributional derivatives ${s^{+}}^{\prime}$ and $s^{{ }^{\prime}}$;

(ii) the analytic derivatives as non-tangential limits of ${s^{+}}^{\prime}(z)$ and $s^{{ }^{\prime}}(z)$ respectively defined in $\mathbb{C}^{+}$and $\mathbb{C}^{-}$through (1.10);

(iii) the inverse Fourier transform derivatives $\left[i \omega \hat{s}^{+}(\omega)\right]^{\vee}$ and $\left[i \omega s^{-}(\omega)\right]^{\vee}$;

(iv) the boundary derivatives

$$
\lim _{z \rightarrow t} \frac{s^{ \pm}(z)-s^{ \pm}(t)}{z-t}, \quad z \rightarrow t \text { in } \mathbb{C}^{ \pm} \text {non - tangentially; }
$$

(v) the classical derivatives of $s^{+}$and $s^{-}$.

When $s \in L_{1}^{2}(\mathbb{R})$, then the first four types of derivatives of $s^{+}$or $s^{-}$as $L^{2}$-functions, always exist and are equal ([6]). The classical derivatives (the type (v)) do not always exist, but when they exist as $L^{2}$-functions they are identical with the former four types. In the sequel we use the notation " " for the first four types of derivatives.

The derivatives defined in Definition 1.1 can be regarded as the (vi) type. The relations between this type and the other types are given in

Proposition 2.1. For $s(t)=\rho(t) e^{i \varphi(t)} \in L_{1}^{2}(\mathbb{R})$, we have

(i)

$$
s^{*}=s^{\prime} \quad \text { and } \quad \rho^{*}=\rho^{\prime}, \quad \text { distributionally; }
$$

(ii) if the classical derivatives $s^{\prime}\left(t_{0}\right), \rho^{\prime}\left(t_{0}\right)$ and $\varphi^{\prime}\left(t_{0}\right)$ all exist, then

$$
\rho^{*}\left(t_{0}\right)=\rho^{\prime}\left(t_{0}\right)
$$

and, if $\rho\left(t_{0}\right) \neq 0$, then

$$
\varphi^{*}\left(t_{0}\right)=\varphi^{\prime}\left(t_{0}\right)
$$

Proof of Property (i) The assertion $s^{*}=s^{\prime}$ is trivial, as $s^{\prime}=s^{+^{\prime}}+s^{-^{\prime}}$ distributionally. Now we prove $\rho^{*}(t)=\rho^{\prime}(t)$. Denote for any $y>0$,

$$
H_{y}(t)=s_{y}^{+}(t)+s_{-y}^{-}(t) \text { and } E_{y}=\left\{t: H_{y}(t) \neq 0\right\} .
$$


Since $E_{y}$ is an open set, it is a union of at most countably many open intervals. In every open interval the function $e^{i \theta_{y}(t)}$ may be smoothly defined through

$$
e^{i \theta_{y}(t)}=\frac{H_{y}(t)}{\left|H_{y}(t)\right|} .
$$

For every $\phi \in \mathfrak{D}$ (the space of smooth functions with compact support on $\mathbb{R}$ ), by applying integration by parts,

$$
\begin{aligned}
& \int_{E_{y}}\left|H_{y}(t)\right| \operatorname{Re}\left\{\frac{s_{y}^{+\prime}(t)+s_{-y}^{-}(t)}{s_{y}^{+}(t)+s_{-y}^{-}(t)}\right\} \phi(t) d t \\
= & \operatorname{Re}\left\{\int_{E_{y}}\left[s_{y}^{+^{\prime}}(t)+s_{-y}^{-{ }^{\prime}}(t)\right] e^{-i \theta_{y}(t)} \phi(t) d t\right\} \\
= & \operatorname{Re}\left\{\left.\left[s_{y}^{+}(t)+s_{-y}^{-}(t)\right] e^{-i \theta_{y}(t)} \phi(t)\right|_{-\infty} ^{+\infty}\right\}-\operatorname{Re}\left\{\int_{E_{y}}\left[s_{y}^{+}(t)+s_{-y}^{-}(t)\right]\left[e^{-i \theta_{y}(t)} \phi(t)\right]^{\prime} d t\right\} \\
= & -\operatorname{Re}\left\{\int_{E_{y}}\left|H_{y}(t)\right| e^{i \theta_{y}(t)}\left[-i \theta_{y}^{\prime}(t) e^{-i \theta_{y}(t)} \phi(t)+e^{-i \theta_{y}(t)} \phi^{\prime}(t)\right] d t\right\} \\
= & -\int_{E_{y}}\left|H_{y}(t)\right| \phi^{\prime}(t) d t .
\end{aligned}
$$

Denote $E=\{t: \rho(t) \neq 0\}$. We have

$$
\lim _{y \rightarrow 0} \chi_{E_{y}}=\chi_{E}, \quad \text { a.e. }
$$

Note that $H_{y}$ is dominated by the sum of the non-tangential maximal functions of $s_{y}^{+}$and $s_{-y}^{-}$ that are both in $L^{2}(\mathbb{R})([9])$. By using Lebesgue's dominated convergence theorem, we have

$$
\int_{-\infty}^{\infty} \rho(t) \operatorname{Re}\left(\frac{s^{+^{\prime}}(t)+s^{-1}(t)}{s^{+}(t)+s^{-}(t)}\right) \phi(t) d t=-\int_{-\infty}^{\infty} \rho(t) \phi^{\prime}(t) d t
$$

that is

$$
\left\langle\rho^{*}(t), \phi(t)\right\rangle=-\left\langle\rho(t), \phi^{\prime}(t)\right\rangle=\left\langle\rho^{\prime}(t), \phi(t)\right\rangle .
$$

So

$$
\rho^{*}=\rho^{\prime}
$$

To show the first assertion of (ii), we consider two cases. The first is $s\left(t_{0}\right)=0$. In the case

$$
\frac{\rho\left(t_{0}+\Delta t\right)-\rho\left(t_{0}\right)}{\Delta t}=\frac{\left|s\left(t_{0}+\Delta t\right)-s\left(t_{0}\right)\right|}{\Delta t} .
$$

Since $\rho^{\prime}\left(t_{0}\right)$ exists, we know that the limit exists as $\Delta t \rightarrow 0$. But, from the right-hand-side we see that when $\Delta t \rightarrow 0+$, the limit $\geq 0$, and when $\Delta t \rightarrow 0-$, the limit $\leq 0$. Therefore, the limit has to be 0 . That is $\rho^{\prime}\left(t_{0}\right)=0=\rho^{*}\left(t_{0}\right)$. The second case is $s\left(t_{0}\right) \neq 0$. Then we have

$$
s^{\prime}\left(t_{0}\right)=\rho^{\prime}\left(t_{0}\right) e^{i \varphi\left(t_{0}\right)}+i \varphi^{\prime}\left(t_{0}\right) \rho\left(t_{0}\right) e^{i \varphi\left(t_{0}\right)} .
$$


Since $\rho\left(t_{0}\right) \neq 0$, we have

$$
\rho\left(t_{0}\right) \operatorname{Re}\left[\frac{s^{\prime}\left(t_{0}\right)}{s\left(t_{0}\right)}\right]=\rho^{\prime}\left(t_{0}\right)=\rho^{*}\left(t_{0}\right)
$$

and

$$
\operatorname{Im}\left[\frac{s^{\prime}\left(t_{0}\right)}{s\left(t_{0}\right)}\right]=\varphi^{\prime}\left(t_{0}\right)=\varphi^{*}\left(t_{0}\right) .
$$

We note that the definitions of H-S derivatives are elaborate that are based on the concepts of Hardy and Sobolev spaces. Once the new type derivatives are defined, the proofs are natural as shown in the following theorems. This shows the necessity of the concepts.

Theorem 2.2. Assume $s(t)=\rho(t) e^{i \varphi(t)} \in L_{1}^{2}(\mathbb{R})$. The mean Fourier frequency defined by (1.3) is identical with

$$
\langle\omega\rangle=\int_{-\infty}^{\infty} \varphi^{*}(t) \rho^{2}(t) d t
$$

where $\varphi^{*}(t)$ is defined in Definition 1.1 .

Proof of Theorem Since $s(t)=\rho(t) e^{i \varphi(t)} \in L_{1}^{2}(\mathbb{R})$, we have $\omega \hat{s}(\omega), \hat{s}(\omega) \in L^{2}(\mathbb{R})$, hence $\langle\omega\rangle$ is well defined. Denote $E=\{t: s(t) \neq 0\}$. With the decomposition $s=s^{+}+s^{-},\left(s^{ \pm}\right)^{\wedge}=$ $\chi_{ \pm} \hat{s}, s^{ \pm}(t)=\rho^{ \pm}(t) e^{i \varphi^{ \pm}(t)}$, we have (also see [6])

$$
\begin{aligned}
\langle\omega\rangle & =\int_{0}^{+\infty} \omega|\hat{s}(\omega)|^{2} d \omega+\int_{-\infty}^{0} \omega|\hat{s}(\omega)|^{2} d \omega \\
& =\operatorname{Im} \int_{-\infty}^{+\infty}\left[s^{+^{\prime}}(t) \overline{s^{+}(t)}+s^{-1}(t) \overline{s^{-}(t)}\right] d t \\
& =\operatorname{Im} \int_{-\infty}^{+\infty}\left[{s^{+}}^{\prime}(t)+s^{-\prime}(t)\right] \overline{\left[s^{+}(t)+s^{-}(t)\right]} d t \\
& =\int_{E} \operatorname{Im}\left\{\frac{s^{+^{\prime}}(t)+s^{-1}(t)}{s^{+}(t)+s^{-}(t)}\right\}\left|s^{+}(t)+s^{-}(t)\right|^{2} d t \\
& =\int_{-\infty}^{+\infty} \varphi^{*}(t) \rho^{2}(t) d t,
\end{aligned}
$$

where the cross terms

$$
\int_{-\infty}^{+\infty} s^{+^{\prime}}(t) \overline{s^{-}(t)} d t \text { and } \int_{-\infty}^{+\infty} s^{-\prime}(t) \overline{s^{+}(t)} d t
$$

vanish because of the Plancherel Theorem and $\chi_{+} \chi_{-}=0$.

There holds a similar formula for $\left\langle\omega^{2}\right\rangle$, as is seen in

Theorem 2.3. Assume $s(t)=\rho(t) e^{i \varphi(t)} \in L_{1}^{2}(\mathbb{R})$. There follows

$$
\begin{aligned}
\left\langle\omega^{2}\right\rangle & \triangleq \int_{-\infty}^{\infty} \omega^{2}|\hat{s}(\omega)|^{2} d \omega \\
& =\int_{-\infty}^{\infty} \rho^{* 2}(t) d t+\int_{-\infty}^{\infty} \varphi^{* 2}(t) \rho^{2}(t) d t
\end{aligned}
$$


Proof of Theorem Since $s(t)=\rho(t) e^{i \varphi(t)} \in L_{1}^{2}(\mathbb{R}), \omega \hat{s}(\omega) \in L^{2}(\mathbb{R})$, and thus $\omega^{2}|\hat{s}(\omega)|^{2} \in$ $L^{1}(\mathbb{R})$. Hence $\left\langle\omega^{2}\right\rangle$ is well defined. With the same notation as in the proof of Theorem 2.2, we have

$$
\begin{aligned}
\left\langle\omega^{2}\right\rangle & =\int_{0}^{+\infty} \omega^{2}|\hat{s}(\omega)|^{2} d \omega+\int_{-\infty}^{0} \omega^{2}|\hat{s}(\omega)|^{2} d \omega \\
& =\int_{-\infty}^{+\infty}\left[s^{+^{\prime}}(t) \overline{{s^{+\prime}}^{\prime}(t)}+{s^{-\prime}}^{\prime}(t) \overline{{s^{-\prime}}^{\prime}(t)}\right] d t \\
& =\int_{-\infty}^{+\infty}\left[{s^{+}}^{\prime}(t)+{s^{-\prime}}^{\prime}(t)\right] \overline{\left[{s^{+}}^{\prime}(t)+s^{-\prime}(t)\right]} d t \\
& =\int_{E}\left|\frac{s^{+^{\prime}}(t)+s^{-\prime}(t)}{s^{+}(t)+s^{-}(t)}\right|^{2}\left|s^{+}(t)+s^{-}(t)\right|^{2} d t \\
& =\int_{E} \operatorname{Re}^{2}\left\{\frac{s^{+^{\prime}}(t)+s^{-\prime}(t)}{s^{+}(t)+s^{-}(t)}\right\}|s(t)|^{2} d t+\int_{E} \operatorname{Im}^{2}\left\{\frac{s^{+^{\prime}}(t)+s^{-\prime}(t)}{s^{+}(t)+s^{-}(t)}\right\}|s(t)|^{2} d t \\
& =\int_{-\infty}^{+\infty} \rho^{* 2}(t) d t+\int_{-\infty}^{+\infty} \varphi^{* 2}(t) \rho^{2}(t) d t .
\end{aligned}
$$

Corollary 2.4. Assume $s(t)=\rho(t) e^{i \varphi(t)} \in L_{1}^{2}(\mathbb{R})$. The bandwidth is

$$
B^{2}=\left\langle\omega^{2}\right\rangle-\langle\omega\rangle^{2}=\int_{-\infty}^{\infty} \rho^{* 2}(t) d t+\int_{-\infty}^{\infty}\left[\varphi^{*}(t)-\langle\omega\rangle\right]^{2} \rho^{2}(t) d t .
$$

Proof of Corollary Based on the expressions of $\langle\omega\rangle,\left\langle\omega^{2}\right\rangle$ obtained in Theorem 2.2 and Theorem 2.3, we easily obtain the result through the equality $B^{2}=\left\langle\omega^{2}\right\rangle-\langle\omega\rangle^{2}$.

Alternatively, the bandwidth can be obtained by following the same steps as in the proof of Theorem 2.3. where

Assume $s(t), t s(t) \in L^{2}(R)$, then $\hat{s}(\omega)=B(\omega) e^{i \psi(\omega)} \in L^{2}(R)$ and $\hat{s}(\omega)=\hat{s}^{+}(\omega)+\hat{s}^{-}(\omega)$,

$$
\hat{s}^{+}(\omega)=\left[\chi_{-} s\right]^{\wedge}(\omega), \quad \hat{s}^{-}(\omega)=\left[\chi_{+} s\right]^{\wedge}(\omega) .
$$

Being similar to the case of the amplitude-phase representations of $s^{ \pm}$, we now have $\hat{s}^{ \pm}(\omega)=$ $B^{ \pm}(\omega) e^{i \psi^{ \pm}(\omega)} \in L^{2}(\mathbb{R})$, where $B^{ \pm}(\omega), B^{ \pm^{\prime}}(\omega)$ and $\psi^{ \pm^{\prime}}(\omega)$ are defined in the same way as (1.8) and (1.7). The H-S derivatives $\hat{s}^{*}(\omega), \psi^{*}(\omega)$ and $B^{*}(\omega)$ are defined as in Definition 1.1.

Definition 2.5. Let $s$ be a square-integrable signal, then we can define the mean of time by

$$
\langle t\rangle \triangleq \int_{-\infty}^{\infty} t|s(t)|^{2} d t
$$

the duration by

$$
T^{2} \triangleq \sigma_{t}^{2} \triangleq \int_{-\infty}^{\infty}(t-\langle t\rangle)^{2}|s(t)|^{2} d t
$$


the mean of any time function $g(t)$ by

$$
\langle g(t)\rangle \triangleq \int_{-\infty}^{\infty} g(t)|s(t)|^{2} d t
$$

and the mean of any Fourier frequency function $h(\omega)$ by

$$
\langle h(\omega)\rangle \triangleq \int_{-\infty}^{\infty} h(\omega)|\hat{s}(\omega)|^{2} d \omega
$$

provided that the right-hand-sides of (2.23), (2.24), (2.25) and (2.26) are well defined integrals. Below we also use the notation

$$
\langle h(\omega)\rangle_{ \pm} \triangleq \int_{-\infty}^{\infty} h(\omega)\left|\hat{s}^{ \pm}(\omega)\right|^{2} d \omega
$$

and

$$
\langle g(t)\rangle_{ \pm} \triangleq \int_{-\infty}^{\infty} g(t)\left|s^{ \pm}(t)\right|^{2} d t
$$

For the mean of time and the duration, we have the corresponding results.

Theorem 2.6. Assume $s(t), t s(t) \in L^{2}(R)$, the mean time defined by (2.23) is identical with

$$
\langle t\rangle=-\int_{-\infty}^{\infty} \psi^{*}(\omega) B^{2}(\omega) d \omega .
$$

Theorem 2.7. Assume $s(t), t s(t) \in L^{2}(R)$, the duration defined by (2.24) is identical with

$$
\sigma_{t}^{2}=\int_{-\infty}^{\infty} B^{* 2}(\omega) d \omega+\int_{-\infty}^{\infty}\left(\psi^{*}(\omega)+\langle t\rangle\right)^{2} B^{2}(\omega) d \omega
$$

The proofs of Theorem 2.6 and Theorem 2.7 are omitted as they are similar to those of Theorem 2.2 and Corollary 2.4.

For a complex-valued signal $s(t)=\rho(t) e^{i \varphi(t)}$, if $s(t), \rho(t)$ and $\varphi(t)$ have the classical derivatives $s^{\prime}(t), \rho^{\prime}(t)$ and $\varphi^{\prime}(t)$, and $s^{\prime}(t), t s(t) \in L^{2}(\mathbb{R})$, then the covariance is defined by

$$
\operatorname{Cov}_{t \omega} \triangleq\left\langle t \varphi^{\prime}(t)\right\rangle-\langle t\rangle\langle\omega\rangle
$$

that is proved to be equal to

$$
-\left\langle\omega \psi^{\prime}(\omega)\right\rangle-\langle t\rangle\langle\omega\rangle
$$

$($ see $[5])$. 
In [6], we extend the definition of covariance to general signal $s(t) \in L_{1}^{2}(\mathbb{R})$ and $t s(t) \in$ $L^{2}(\mathbb{R})$ by

$$
\operatorname{Cov}_{t \omega} \triangleq\left\langle t \varphi^{+^{\prime}}(t)\right\rangle_{+}+\left\langle t \varphi^{-\prime}(t)\right\rangle_{-}-\langle t\rangle\langle\omega\rangle
$$

and we show that $\mathrm{Cov}_{\mathrm{t} \omega}$ has an alternative formula

$$
\operatorname{Cov}_{\mathrm{t} \omega}=-\left\langle\omega \psi^{+^{\prime}}(\omega)\right\rangle_{+}-\left\langle\omega \psi^{-\prime}(\omega)\right\rangle_{-}-\langle t\rangle\langle\omega\rangle .
$$

By employing the H-S derivatives, we can reduce the formula of the covariance (2.32) to the same form as (2.31).

Theorem 2.8. Assume $s(t)=\rho(t) e^{i \varphi(t)} \in L_{1}^{2}(\mathbb{R})$ and $t s(t) \in L^{2}(\mathbb{R})$, there follows

$$
\operatorname{Cov}_{t \omega}=\left\langle t \varphi^{*}(t)\right\rangle-\langle t\rangle\langle\omega\rangle=-\left\langle\omega \psi^{*}(\omega)\right\rangle-\langle t\rangle\langle\omega\rangle \text {. }
$$

Proof of Theorem With the decomposition $s=s^{+}+s^{-},\left(s^{ \pm}\right)^{\wedge}=\chi_{ \pm} \hat{s}, s^{ \pm}(t)=\rho^{ \pm}(t) e^{i \varphi^{ \pm}(t)}$, we proved in [6] that

$$
\begin{aligned}
\left\langle t \varphi^{+^{\prime}}(t)\right\rangle_{+}+\left\langle t \varphi^{-^{\prime}}(t)\right\rangle_{-} & =\int_{-\infty}^{\infty} t{\varphi^{+}}^{\prime}(t)\left|s^{+}(t)\right|^{2} d t+\int_{-\infty}^{\infty} t \varphi^{-\prime}(t)\left|s^{-}(t)\right|^{2} d t \\
& =\operatorname{Im} \int_{-\infty}^{\infty}\left[s^{+^{\prime}}(t) \overline{t s^{+}(t)}+s^{-\prime}(t) \overline{t s^{-}(t)}\right] d t .
\end{aligned}
$$

Denote $E=\{t: s(t) \neq 0\}$,

$$
\begin{aligned}
& \operatorname{Im} \int_{-\infty}^{\infty}\left[s^{+^{\prime}}(t) \overline{t s^{+}(t)}+s^{-^{\prime}}(t) \overline{t s^{-}(t)}\right] d t \\
= & \operatorname{Im} \int_{-\infty}^{\infty}\left[s^{+^{\prime}}(t)+s^{-^{\prime}}(t)\right] \overline{\left[t s^{+}(t)+t s^{-}(t)\right]} d t \\
= & \operatorname{Im} \int_{E} \frac{s^{+^{\prime}}(t)+s^{-\prime}(t)}{s^{+}(t)+s^{-}(t)} t|s(t)|^{2} d t \\
= & \int_{-\infty}^{\infty} t \varphi^{*}(t)|s(t)|^{2} d t \\
= & \left\langle t \varphi^{*}(t)\right\rangle,
\end{aligned}
$$

where the cross terms

$$
\int_{-\infty}^{\infty} s^{+^{\prime}}(t) \overline{t s^{-}(t)} d t \text { and } \int_{-\infty}^{\infty} s^{-^{\prime}}(t) \overline{t s^{+}(t)} d t
$$

equal to zero because of the Parseval equality.

Similarly we have

$$
-\left\langle\omega \psi^{+^{\prime}}(\omega)\right\rangle_{+}-\left\langle\omega \psi^{-\prime}(\omega)\right\rangle_{-}=-\left\langle\omega \psi^{*}(\omega)\right\rangle .
$$


For higher moments of Fourier frequency $\left\langle\omega^{n}\right\rangle$ we have representations of the same kind.

We have prove in $[6]$ that if $s(t) \in L_{n}^{2}(\mathbb{R})$, then $s^{ \pm(n)}(z) \in H^{2}\left(\mathbb{C}^{ \pm}\right)$, and $s^{ \pm(n)}(t)$ are defined to be the non-tangential boundary limits of $s^{ \pm(n)}(z)$. In this case we define the $n$-th H-S derivative of $s(t)$ by

$$
s^{(n)^{*}}(t) \triangleq s^{+(n)}(t)+s^{-(n)}(t) .
$$

If $s(t) \in L^{2}(\mathbb{R})$ and $t^{n} s(t) \in L^{2}(\mathbb{R})$, then we can define the $n$-th H-S derivative of $\hat{s}(\omega)$ by

$$
\hat{s}^{(n)^{*}}(\omega) \triangleq\left(\hat{s}^{+}\right)^{(n)}(\omega)+\left(\hat{s}^{-}\right)^{(n)}(\omega),
$$

where $\left(\hat{s}^{ \pm}\right)^{(n)}(\omega)$ are the non-tangential boundary limits of $\left(\hat{s}^{ \pm}\right)^{(n)}(z)$.

With similar techniques we obtain

Theorem 2.9. Assume $s(t) \in L_{n}^{2}(\mathbb{R})$, there follow

$$
\begin{aligned}
\left\langle\omega^{2 k}\right\rangle & =\int_{-\infty}^{\infty}\left|s^{(k)^{*}}(t)\right|^{2} d t \\
\left\langle\omega^{2 k-1}\right\rangle & =\int_{-\infty}^{\infty} \operatorname{Im}\left[s^{(k)^{*}}(t) \overline{s^{(k-1)^{*}}(t)}\right] d t, \quad k=1, \ldots, n .
\end{aligned}
$$

Theorem 2.10. Assume $s(t) \in L^{2}(\mathbb{R})$ and $t^{n} s(t) \in L^{2}(\mathbb{R})$, there follow

$$
\begin{aligned}
\left\langle t^{2 k}\right\rangle & =\int_{-\infty}^{\infty}\left|\hat{s}^{(k)^{*}}(\omega)\right|^{2} d \omega \\
\left\langle t^{2 k-1}\right\rangle & =-\int_{-\infty}^{\infty} \operatorname{Im}\left[\hat{s}^{(k)^{*}}(\omega) \overline{\hat{s}^{(k-1)^{*}}(\omega)}\right] d \omega, \quad k=1, \ldots, n .
\end{aligned}
$$

\section{Mean Frequency and Time for Period Signal and Infinite Discrete Signal}

For $s\left(e^{i t}\right)=\rho(t) e^{i \varphi(t)}, t \in[0,2 \pi)$, if we assume that $s\left(e^{i t}\right) \in L_{1}^{2}([0,2 \pi))$, we can also define the Hardy-Sobolev derivatives related to $s\left(e^{i t}\right)$ in a similar way. $s\left(e^{i t}\right) \in L^{2}([0,2 \pi))$ may be expanded into its Fourier series convergent in the $L^{2}$-norm sense

$$
s\left(e^{i t}\right)=\frac{1}{\sqrt{2 \pi}} \sum_{k=-\infty}^{\infty} c_{k} e^{i k t},
$$

where $c_{k}$ 's are the Fourier coefficients,

$$
c_{k}=\frac{1}{\sqrt{2 \pi}} \int_{0}^{2 \pi} s\left(e^{i t}\right) e^{-i k t} d t, \quad k=0, \pm 1, \pm 2, \cdots,
$$

and There holds the Parseval equality

$$
\|s\|_{2}^{2}=\sum_{k=-\infty}^{\infty}\left|c_{k}\right|^{2} .
$$


Now we decompose $s\left(e^{i t}\right)$ into two parts $s^{+}\left(e^{i t}\right)$ and $s^{-}\left(e^{i t}\right)$, where $s^{ \pm}\left(e^{i t}\right)=\frac{1}{2}(s \pm i \tilde{H} s \pm$ $\left.c_{0}\right)$, which are the non-tangential boundary values of the analytic functions $s^{+}(z)$ and $s^{-}(z)$, respectively, where

$$
s^{+}(z)=\frac{1}{2 \pi i} \int_{\partial \mathbb{D}} \frac{s(\zeta)}{\zeta-z} d \zeta=\frac{1}{\sqrt{2 \pi}} \sum_{k=0}^{+\infty} c_{k} z^{k}, \quad z \in \mathbb{D},
$$

and

$$
s^{-}(z)=-\frac{1}{2 \pi i} \int_{\partial \mathbb{D}} \frac{s(\zeta)}{\zeta-z} d \zeta=\frac{1}{\sqrt{2 \pi}} \sum_{k=-\infty}^{-1} c_{k} z^{k}, \quad z \in \mathbb{C} \backslash \overline{\mathbb{D}}
$$

and $\tilde{H}$ is the circular Hilbert transformation given by

$$
\tilde{H} f=\frac{1}{2 \pi} p \cdot v \cdot \int_{-\pi}^{\pi} \cot \left(\frac{t-s}{2}\right) f(s) d s, \text { a.e. }
$$

Since $s\left(e^{i t}\right) \in L_{1}^{2}([0,2 \pi))$, then $s^{+^{\prime}}\left(e^{i t}\right)$ and $s^{{ }^{\prime}}\left(e^{i t}\right)$ all exist as $L^{2}$-functions on $\partial \mathbb{D}$ and are the non-tangential boundary values of $s^{+^{\prime}}(z)$ and $s^{-\prime}(z)$, respectively (see [6]).

Based on the above decomposition, we introduce the Hardy-Sobolev derivatives related to $s\left(e^{i t}\right)$.

Definition 3.1. If $s\left(e^{i t}\right)=\rho(t) e^{i \varphi(t)} \in L_{1}^{2}(\mathbb{R})$, then we define the Hardy-Sobolev derivatives of $s\left(e^{i t}\right), \rho(t)$ and $\varphi(t)$ by, respectively,

$$
\begin{gathered}
s^{*}\left(e^{i t}\right)=s^{+^{\prime}}\left(e^{i t}\right)+s^{{ }^{\prime}}\left(e^{i t}\right), \\
\rho^{*}(t)= \begin{cases}-\rho(t) \operatorname{Im}\left[e^{i t} \frac{s^{{ }^{\prime}}\left(e^{i t}\right)+s^{-\prime}\left(e^{i t}\right)}{s^{+}\left(e^{i t}\right)+s^{-}\left(e^{i t}\right)}\right] & \text { if } s\left(e^{i t}\right) \neq 0, \\
0 & \text { if } s\left(e^{i t}\right)=0,\end{cases}
\end{gathered}
$$

and

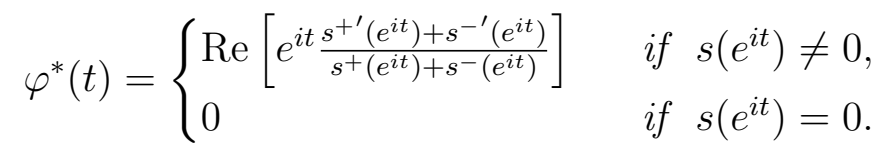

It is obvious that $s^{*}, \rho^{*} \in L^{2}(\mathbb{R})$ and $\varphi^{*}$ is measurable. Furthermore, assume $s\left(e^{i t}\right) \in$ $L_{m}^{2}([0,2 \pi))$, then we can define the $n$-th Hardy-Sobolev derivative of $s\left(e^{i t}\right)$, namely,

$$
s^{(n)^{*}}\left(e^{i t}\right)=s^{+(n)}\left(e^{i t}\right)+s^{-(n)}\left(e^{i t}\right), \quad n=1, \ldots, m .
$$

For periodic signal $s\left(e^{i t}\right)$, the corresponding mean of the Fourier frequency and higher moments of the Fourier frequency are given by:

$$
\langle k\rangle \triangleq \sum_{k=-\infty}^{\infty} k\left|c_{k}\right|^{2} \quad \text { and } \quad\left\langle k^{n}\right\rangle \triangleq \sum_{k=-\infty}^{\infty} k^{n}\left|c_{k}\right|^{2}, \quad n \in \mathbf{Z}^{+} .
$$

Then we can prove 
Theorem 3.2. Assume $s\left(e^{i t}\right)=\rho(t) e^{i \varphi(t)} \in L_{1}^{2}([0,2 \pi))$. There hold

$$
\begin{aligned}
\langle k\rangle & =\int_{0}^{2 \pi} \varphi^{*}(t) \rho^{2}(t) d t \\
\left\langle k^{2}\right\rangle & =\int_{0}^{2 \pi} \rho^{* 2}(t) d t+\int_{0}^{2 \pi} \varphi^{* 2}(t) \rho^{2}(t) d t,
\end{aligned}
$$

where $\varphi^{*}(t)$ are defined in the Definition 3.1. In general, if $s\left(e^{i t}\right)=\rho(t) e^{i \varphi(t)} \in L_{m}^{2}([0,2 \pi))$, then

$$
\begin{aligned}
& \left\langle k^{2 n-1}\right\rangle=\int_{0}^{2 \pi} \operatorname{Re}\left[e^{i t} s^{(n)^{*}}\left(e^{i t}\right) \overline{s^{(n-1)^{*}}\left(e^{i t}\right)}\right] d t, \\
& \left\langle k^{2 n}\right\rangle=\int_{0}^{2 \pi}\left|s^{(n)^{*}}\left(e^{i t}\right)\right|^{2} d t, \quad n=1, \ldots, m .
\end{aligned}
$$

Proof of Theorem Since $s\left(e^{i t}\right) \in L_{1}^{2}([0,2 \pi))$, then in the $L^{2}$-norm sense $s^{+}\left(e^{i t}\right)=\frac{1}{\sqrt{2 \pi}} \sum_{k=0}^{\infty} c_{k} e^{i k t}$ and $s^{-}\left(e^{i t}\right)=\frac{1}{\sqrt{2 \pi}} \sum_{k=-\infty}^{-1} c_{k} e^{i k t}$, where $c_{k}$ 's are the Fourier coefficients of $s\left(e^{i t}\right)$. Thus $e^{i t} s^{+^{\prime}}\left(e^{i t}\right)=$ $\frac{1}{\sqrt{2 \pi}} \sum_{k=0}^{\infty} k c_{k} e^{i k t}$ and $e^{i t} s^{-\prime}\left(e^{i t}\right)=\frac{1}{\sqrt{2 \pi}} \sum_{k=-\infty}^{-1} k c_{k} e^{i k t}$. Assume $E=\left\{t \in[0,2 \pi) \mid s\left(e^{i t}\right) \neq 0\right\}$. By the Parseval equality, We have

$$
\begin{aligned}
\langle k\rangle & =\sum_{k=0}^{\infty} k c_{k} \overline{c_{k}}+\sum_{k=-\infty}^{-1} k c_{k} \overline{c_{k}} \\
& =\int_{0}^{2 \pi} e^{i t} s^{+^{\prime}}\left(e^{i t}\right) \overline{s^{+}\left(e^{i t}\right)} d t+\int_{0}^{2 \pi} e^{i t} s^{-\prime}\left(e^{i t}\right) \overline{s^{-}\left(e^{i t}\right)} d t \\
& =\int_{0}^{2 \pi} e^{i t}\left[{s^{+}}^{\prime}\left(e^{i t}\right)+s^{-\prime}\left(e^{i t}\right)\right] \overline{\left[s^{+}\left(e^{i t}\right)+s^{-}\left(e^{i t}\right)\right]} d t \\
& =\int_{E} e^{i t} \frac{s^{*}\left(e^{i t}\right)}{s\left(e^{i t}\right)}\left|s\left(e^{i t}\right)\right|^{2} d t \\
& =\int_{E} \operatorname{Re}\left\{e^{i t} \frac{s^{*}\left(e^{i t}\right)}{s\left(e^{i t}\right)}\right\}\left|s\left(e^{i t}\right)\right|^{2} d t \\
& =\int_{0}^{2 \pi} \varphi^{*}(t)\left|s\left(e^{i t}\right)\right|^{2} d t,
\end{aligned}
$$

where the cross terms

$$
\int_{0}^{2 \pi} e^{i t} s^{+^{\prime}}\left(e^{i t}\right) \overline{s^{-}\left(e^{i t}\right)} d t \text { and } \int_{0}^{2 \pi} e^{i t} s^{-\prime}\left(e^{i t}\right) \overline{s^{+}\left(e^{i t}\right)} d t
$$


equal to zero because of the orthogonality.

$$
\begin{aligned}
& \left\langle k^{2}\right\rangle=\sum_{k=0}^{\infty} k c_{k} \overline{k c_{k}}+\sum_{k=-\infty}^{-1} k c_{k} \overline{k c_{k}} \\
& =\int_{0}^{2 \pi} e^{i t} s^{+^{\prime}}\left(e^{i t}\right) \overline{e^{i t} s^{+^{\prime}}\left(e^{i t}\right)} d t+\int_{0}^{2 \pi} e^{i t} s^{-\prime}\left(e^{i t}\right) \overline{e^{i t} s^{-\prime}\left(e^{i t}\right)} d t \\
& =\int_{0}^{2 \pi} e^{i t}\left[s^{+^{\prime}}\left(e^{i t}\right)+s^{-^{\prime}}\left(e^{i t}\right)\right] \overline{\left.\left.e^{i t}\left[{s^{+^{\prime}}}^{(i t}\right)+{s^{-\prime}}^{(i t}\right)\right]} d t \\
& =\int_{E} \frac{\left|e^{i t} s^{*}\left(e^{i t}\right)\right|^{2}}{\left|s\left(e^{i t}\right)\right|^{2}}\left|s\left(e^{i t}\right)\right|^{2} d t \\
& =\int_{E} \operatorname{Re}^{2}\left\{e^{i t} \frac{s^{*}\left(e^{i t}\right)}{s\left(e^{i t}\right)}\right\}\left|s\left(e^{i t}\right)\right|^{2} d t+\int_{E} \operatorname{Im}^{2}\left\{e^{i t} \frac{s^{*}\left(e^{i t}\right)}{s\left(e^{i t}\right)}\right\}\left|s\left(e^{i t}\right)\right|^{2} d t \\
& =\int_{0}^{2 \pi} \varphi^{* 2}(t)\left|s\left(e^{i t}\right)\right|^{2} d t+\int_{0}^{2 \pi} \rho^{* 2}(t) d t .
\end{aligned}
$$

The proofs of the relations (3.38) and (3.37) are similar and thus omitted.

Next we study $\langle t\rangle$ and the related variance. Since $t$ in $s\left(e^{i t}\right)$ belongs to the interval $[0,2 \pi)$, $t$ is treated as an angular random variable under the radian measure, and the energy density function of $t$ is $\left|s\left(e^{i t}\right)\right|^{2}$ (see [15]). The mean angle (direction) of $t$ is to be defined by

$$
\langle t\rangle=\arg E\left(e^{i t}\right)=\arg \int_{0}^{2 \pi} e^{i t}\left|s\left(e^{i t}\right)\right|^{2} d t,
$$

and the circular variance of $t$ by

$$
V_{t}=1-E \cos (t-\langle t\rangle)=1-\int_{0}^{2 \pi} \cos (t-\langle t\rangle)\left|s\left(e^{i t}\right)\right|^{2} d t
$$

We have

$$
\begin{aligned}
\int_{0}^{2 \pi} \cos (t-\langle t\rangle)\left|s\left(e^{i t}\right)\right|^{2} d t & =\operatorname{Re}\left\{\int_{0}^{2 \pi} e^{i(t-\langle t\rangle)}\left|s\left(e^{i t}\right)\right|^{2} d t\right\} \\
& =\operatorname{Re}\left\{\int_{0}^{2 \pi} e^{i t}\left|s\left(e^{i t}\right)\right|^{2} d t \frac{\left.\left|\int_{0}^{2 \pi} e^{i t}\right| s\left(e^{i t}\right)\right|^{2} d t \mid}{\int_{0}^{2 \pi} e^{i t}\left|s\left(e^{i t}\right)\right|^{2} d t}\right\} \\
& =\left.\left|\int_{0}^{2 \pi} e^{i t}\right| s\left(e^{i t}\right)\right|^{2} d t \mid
\end{aligned}
$$

Hence

$$
V_{t}=1-\left.\left|\int_{0}^{2 \pi} e^{i t}\right| s\left(e^{i t}\right)\right|^{2} d t \mid
$$

If we consider the mean time of the period signal $s\left(e^{i t}\right)$ like (2.29), then the major difficulty would be the replacement of "phase derivative" of $\left\{c_{k}\right\}$. The central difference, forward and 
backward differences are all used in literature for discrete phase derivative (see [2], [3], [4], [21]). In the present paper, with $c_{k}=\left|c_{k}\right| e^{i \psi(k)}$ where $\psi(k)$ is phase function of $c_{k}$, we define the discrete-time phase derivative as the backward difference of the phase:

$$
\psi^{*}(k)=[\psi(k)-\psi(k-1)] \bmod 2 \pi .
$$

Since $\psi^{*}(k)$ belongs to the interval $[0,2 \pi)$, we can take $\psi^{*}(k)$ as an angular random variable and its density function is $\left|c_{k}\right|^{2}$. Thus we can define the mean value and variance of $\psi^{*}(k)$ as

$$
\left\langle\psi^{*}(k)\right\rangle=\arg \left[\sum_{k=-\infty}^{+\infty} e^{i \psi^{*}(k)}\left|c_{k}\right|^{2}\right]
$$

and

$$
V_{\psi^{*}(k)}=1-\sum_{k=-\infty}^{+\infty} \cos \left[\psi^{*}(k)-\left\langle\psi^{*}(k)\right\rangle\right]\left|c_{k}\right|^{2}=1-\left.\left|\sum_{k=-\infty}^{+\infty} e^{i \psi^{*}(k)}\right| c_{k}\right|^{2} \mid .
$$

We have the following theorem:

Theorem 3.3. Assume $s\left(e^{i t}\right)=\rho(t) e^{i \varphi(t)} \in L_{1}^{2}([0,2 \pi))$. There holds

$$
\langle t\rangle \triangleq \arg \left[\int_{0}^{2 \pi} e^{i t}\left|s\left(e^{i t}\right)\right|^{2} d t\right]=\arg \left[\sum_{k=-\infty}^{\prime \infty} e^{-i \psi^{*}(k)}\left|c_{k}\right|^{2}\left|\frac{c_{k-1}}{c_{k}}\right|\right]
$$

where $\left\{c_{k}\right\}$ are the Fourier coefficients of $s\left(e^{i t}\right)$, and $\sum^{\prime}$ is the summation over the indices $k^{\prime} s$ at which $c_{k} \neq 0$.

Proof of Theorem Since $s\left(e^{i t}\right) \in L_{1}^{2}([0,2 \pi))$, then $\left\{c_{n}\right\} \in l^{2}$, and thus $\left\{c_{n-1} \overline{c_{n}}\right\} \in l^{1}$ by the Hölder inequality. By the Parseval equality we have

$$
\begin{aligned}
\langle t\rangle & \triangleq \arg \left[\int_{0}^{2 \pi} e^{i t}\left|s\left(e^{i t}\right)\right|^{2} d t\right] \\
& =\arg \left[\int_{0}^{2 \pi} e^{i t} s\left(e^{i t}\right) \overline{s\left(e^{i t}\right)} d t\right] \\
& =\arg \left[\sum_{k=-\infty}^{\infty} c_{k-1} \overline{c_{k}}\right] \\
& =\arg \left[\sum_{k=-\infty}^{\infty}\left|c_{k-1}\right| e^{i \psi(k-1)}\left|c_{k}\right| e^{-i \psi(k)}\right] \\
& =\arg \left[\sum_{k=-\infty}^{\infty} e^{-i \psi^{*}(k)}\left|c_{k}\right|^{2}\left|\frac{c_{k-1}}{c_{k}}\right|\right]
\end{aligned}
$$

Through (3.42) we obtain easily

$$
\left\langle-\psi^{*}(k)\right\rangle=\arg \left[\sum_{k=-\infty}^{+\infty} e^{-i \psi^{*}(k)}\left|c_{k}\right|^{2}\right],
$$


Comparing (3.44) to (3.45), we find that the mean of time $t$ is different from the mean of group delay of $s\left(e^{i t}\right)$, that is $-\psi^{*}(n)$. But in the continuous case the mean of time of the signal is always equal to the mean of the group delay of the signal (see (2.29)).

From Theorem 3.3, we obtain another representation of the circular variance of time $t$ :

$$
V_{t}=1-\left.\left|\int_{0}^{2 \pi} e^{i t}\right| s\left(e^{i t}\right)\right|^{2} d t|=1-| \sum_{k=-\infty}^{\infty} e^{-i \psi^{*}(k)}\left|c_{k}\right|^{2} \mid \frac{c_{k-1}}{c_{k}} \| .
$$

We also have the circular variance of $-\psi^{*}(n)$ through (3.43)

$$
V_{-\psi^{*}(k)}=1-\sum_{k=-\infty}^{+\infty} \cos \left[-\psi^{*}(k)-\left\langle\psi^{*}(k)\right\rangle\right]\left|c_{k}\right|^{2}=1-\left.\left|\sum_{k=-\infty}^{+\infty} e^{-i \psi^{*}(k)}\right| c_{k}\right|^{2} \mid .
$$

By comparing (3.46) with (3.47), we have

$$
V_{t} \neq V_{-\psi^{*}(k)}
$$

This, however, is different from the continuous case given by (2.30), as in the latter the variance of time is always greater than the variance of group delay of the signal, provided that the signal is not a zero signal. Note that Hardy spaces and H-S derivatives are not concerned in the discrete signals case, because no technical problems motivate the related decomposition.

Now consider discrete signals $\{x(n)\}_{n=-\infty}^{+\infty}$. Their discrete-time Fourier transform, also called frequency spectrum, is the restriction of their $Z$-transform to the unit circle. They are the complex-valued periodic functions (see [16], [12]),

$$
X\left(e^{i \omega}\right)=\sum_{n=-\infty}^{+\infty} x(n) e^{-i n \omega}
$$

There is an inversion transformation to get back the sequence:

$$
x(n)=\frac{1}{2 \pi} \int_{0}^{2 \pi} X\left(e^{i \omega}\right) e^{i \omega n} d \omega .
$$

The discrete signals and the periodic signals are dual to each other in the Fourier transform sense. So the mean of time and frequency for a discrete signal can be converted to the mean of frequency and time of the corresponding periodic signal, thus we omit the details for discrete signals.

Some studies treat discrete signals, especially, real-valued signals, through their associated analytic signals defined by

$$
z(n)=x(n)+i H_{d} x(n),
$$

where $\{x(n)\}_{n=-\infty}^{+\infty}$ is the discrete signal, and $H_{d}$ is the discrete Hilbert transform for infinite discrete signals, defined by

$$
H_{d} x(n)=\sum_{m=-\infty}^{+\infty} h(n-m) x(m)
$$


where

$$
h(n)= \begin{cases}\frac{2}{\pi} \frac{\sin ^{2}\left(\pi \frac{n}{2}\right)}{n} & \text { if } n \neq 0 \\ 0 & \text { if } n=0 .\end{cases}
$$

After getting the associated analytic signals one applies similar analysis as above to $Z(n)$.

\section{Mean Frequency and Time for Periodic Discrete signal}

In this section we study periodic discrete signals, or equivalently, finite discrete signals $\{x(n)\}_{n=0}^{N-1}([21])$. We first define the associated discrete analytic signals $z(n)$, by

$$
z(n)=x(n)+i \tilde{H}_{d} x(n),
$$

where $\tilde{H}_{d}$ is the discrete Hilbert transformation for finite discrete signals defined through (see [10])

$$
\tilde{H}_{d} x(n)=\sum_{m=0}^{N-1} h(n-m) x(m)
$$

where

$$
h(n)= \begin{cases}2 \sin ^{2}(\pi n / 2) \cot (\pi n / N) & n=1,2, \cdots, N-1 ; \\ 0 & n=0 .\end{cases}
$$

The discrete Fourier transform of $x(n)$ is given by

$$
X(k)=\frac{1}{N} \sum_{n=0}^{N-1} x(n) e^{-i \frac{2 \pi n k}{N}},
$$

and $\{x(n)\}$ is represented as

$$
x(n)=\sum_{k=0}^{N-1} X(k) e^{i \frac{2 \pi n k}{N}} .
$$

The discrete analytic signal $z(n)$, defined by $(4.51)$ and its DFT, $Z(k)$, are both finite signals of period $\mathrm{N}$, where $Z(k)$ is given by

$$
Z(k)=\frac{1}{N} \sum_{n=0}^{N-1} z(n) e^{-i \frac{2 \pi n k}{N}} .
$$

Let $z(n)=|z(n)| e^{i \phi(n)}$, where $\phi(n)=\arg [z(n)]$. We define the discrete phase derivative of $\{x(n)\}$ by the back difference of the phase $\phi(n)$ :

$$
\phi^{*}(n)=[\phi(n)-\phi(n-1)] \bmod 2 \pi .
$$

We take $\{2 \pi k / N\}_{k=0}^{N-1}$ and $\left\{\phi^{*}(n)\right\}$ as discrete angular random variables, then the mean value and variance of $\{2 \pi k / N\}_{k=0}^{N-1}$ can be similarly defined like (3.39) and (3.40) as follows (see $[15])$

$$
\langle 2 \pi k / N\rangle=\arg \left[\sum_{k=0}^{N-1} e^{i 2 \pi k / N}|Z(k)|^{2}\right]
$$


and

$$
\begin{aligned}
V_{2 \pi k / N} & =1-E \cos (2 \pi k / N-\langle 2 \pi k / N\rangle) \\
& =1-\sum_{k=0}^{N-1} \cos (2 \pi k / N-\langle 2 \pi k / N\rangle)|Z(k)|^{2} \\
& =1-\left.\left|\sum_{k=0}^{N-1} e^{i 2 \pi k / N}\right| Z(k)\right|^{2} \mid .
\end{aligned}
$$

The above equations (4.53) and (4.54) a re the discrete counterpart of (1.3) and (2.21). As in the Theorem 3.3, further writing out (4.53) and (4.54) in the discrete time domain yield

$$
\langle 2 \pi k / N\rangle=\arg \left[\sum_{n=0}^{\prime N-1} e^{i \phi^{*}(n)}|z(n)|^{2}\left|\frac{z(n-1)}{z(n)}\right|\right] .
$$

and

$$
V_{2 \pi k / N}=1-\left.\left|\sum_{n=0}^{\prime N-1} e^{i \phi^{*}(n)}\right| z(n)\right|^{2} \mid \frac{z(n-1)}{z(n)} \| .
$$

The mean and variance of phase derivative $\phi^{*}(n)$ are given, respectively, by

$$
\left\langle\phi^{*}(n)\right\rangle=\arg \left[\sum_{n=0}^{N-1} e^{i \phi^{*}(n)}|z(n)|^{2}\right],
$$

and

$$
V_{\phi^{*}(n)}=1-\left.\left|\sum_{n=0}^{N-1} e^{i \phi^{*}(n)}\right| z(n)\right|^{2} \mid .
$$

The relations (4.55), (4.57),(4.56) and (4.58) show that the results for the finite discrete signals are similar to those for the infinite discrete signals.

\section{References}

[1] P. Auscher, E. Russ, Ph. Tchamitchian, Hardy Sobolev spaces on strong Lipchitz domains in $\mathbb{R}^{n}$, J. Funct. Anal. 218 (2005) 54-109.

[2] B. Boashash, G. Jones, P.O'Shea, Instantaneous frequency of signals: Concepts, estimation techniques, and applications, Proc. SPIE Int. Soc. Opt. Eng. 1152 (1989) 382-399.

[3] B. Boashash, P.O'Shea, M.J. Arnold, Algorithms for instantaneous frequency estimation: A comparative study, Proc. SPIE Int. Soc. Opt. Eng. 1348 (1989).

[4] T.A.C.M. Claasen, W.F G. Mecklenbrauker, The Wigner distribution-a tool for timefrequency signal analysis, parts I, II and III, Philips J. Res. 35 (1980) 217-250, 276-300, and 372-389. 
[5] L. Cohen, Time-Frequency Analysis: Theory and Applications, Prentice Hall, 1995.

[6] P. Dang, T. Qian, Z. You, Hardy-Sobolev spaces decomposition in signal analysis, J. Fourier. Anal. Appl., http://www.springerlink.com/content/ku0g747146k84113/, accepted on 19 April 2010, DOI: 10.1007/s00041-010-9132-7.

[7] L.M. Fink, Relations between the spectrum and instantaneous frequency of a signal, Translated in: Problems Inform. Transm. 2 (1966) 11-21.

[8] D. Gabor, Theory of communication, J. IEE. 93(III) (1946) 429-457.

[9] J.B. Garnett, Bounded Analytic Functions, Academic Press, 1987.

[10] S.L. Hahn, Hilbert Transforms in Signal Processing, Artech House, 1996.

[11] N.E. Huang et al, The empirical mode decomposition and the Hilbert spectrum for nonlinear and non-stationary time series analysis, Proc. R. Soc. London A. 454 (1998) 903-995.

[12] H. Li, L.Q. Li, T. Qian, Discrete-time analytic signals and Bedrosian product theorems, Digital Siganl Process. 20(2010) 982-990.

[13] Z. Lou, A. McIntosh, Hardy spaces of exact form on $\mathbb{R}^{n}$, Trans. Amer. Math. Soc. 357 (2005) 1469-1496.

[14] L. Mandel, Interpretation of instantaneous frequencies, Amer. Jour. Phys. 42 (1974) 840846.

[15] K.V. Mardia, Statistics of Directional Data, London: Academic, 1972.

[16] A.V. Oppenheim, R.W. Schafer, J.R. Buck, Discrete-Time Signal Processing, PrenticeHall, Englewood Cliffs, NJ, 1999.

[17] B. Picinbono, On instantaneous amplitude and phase of signals, IEEE Transactions on Signal Processing, 45 (1997) 552-560.

[18] T. Qian, Mono-components for decomposition of signals, Math. Meth. Appl. Sci. 29 (2006) 1187-1198.

[19] T. Qian, Phase derivative of Nevanlinna functions and applications, Math. Meth. Appl. Sci. 32 (2009) 253-263.

[20] E.M. Stein, Singular Integrals and Differentiability Properties of Functions, Princeton University Press, Princeton, New Jersey, 1970.

[21] M.G. Sun, R.J. Sclabassi, Discrete-time instantaneous frequency and its computation, IEEE Transactions on Signal Processing, 41(1993) 1867-1880. 